\title{
Analysis of shear wall structure using optimal membrane triangle element
}

\begin{abstract}
In this study an alternate formulation, using optimal membrane triangle elements in finite element (FE) programming has been implemented. The formulation showed that more efficient computation was achieved and the accuracy of the FE program was established using some standard benchmark examples. Numerical studies indicate that the FE idealization, with coarse mesh using this alternative optimal membrane triangle element, produced good results for the analysis of shear wall structures. The results were found to be satisfactory with a wide range of element aspect ratio.
\end{abstract}

Keyword: Finite elements, High performance element, Drilling degrees of freedom 